BULLETIN (New Series) OF THE

AMERICAN MATHEMATICAL SOCIETY

Volume 37, Number 4, Pages 407-436

S 0273-0979(00)00881-8

Article electronically published on June 26, 2000

\title{
MATHEMATICAL PROBLEMS
}

\author{
DAVID HILBERT
}

Lecture delivered before the International Congress of Mathematicians at Paris in 1900.

Who of us would not be glad to lift the veil behind which the future lies hidden; to cast a glance at the next advances of our science and at the secrets of its development during future centuries? What particular goals will there be toward which the leading mathematical spirits of coming generations will strive? What new methods and new facts in the wide and rich field of mathematical thought will the new centuries disclose?

History teaches the continuity of the development of science. We know that every age has its own problems, which the following age either solves or casts aside as profitless and replaces by new ones. If we would obtain an idea of the probable development of mathematical knowledge in the immediate future, we must let the unsettled questions pass before our minds and look over the problems which the science of to-day sets and whose solution we expect from the future. To such a review of problems the present day, lying at the meeting of the centuries, seems to me well adapted. For the close of a great epoch not only invites us to look back into the past but also directs our thoughts to the unknown future.

The deep significance of certain problems for the advance of mathematical science in general and the important rôle which they play in the work of the individual investigator are not to be denied. As long as a branch of science offers an abundance of problems, so long is it alive; a lack of problems foreshadows extinction or the cessation of independent development. Just as every human undertaking pursues certain objects, so also mathematical research requires its problems. It is by the solution of problems that the investigator tests the temper of his steel; he finds new methods and new outlooks, and gains a wider and freer horizon.

It is difficult and often impossible to judge the value of a problem correctly in advance; for the final award depends upon the grain which science obtains from the problem. Nevertheless we can ask whether there are general criteria which mark a good mathematical problem. An old French mathematician said: "A mathematical theory is not to be considered complete until you have made it so clear that you can explain it to the first man whom you meet on the street." This clearness and ease of comprehension, here insisted on for a mathematical theory, I should still more demand for a mathematical problem if it is to be perfect; for what is clear and easily comprehended attracts, the complicated repels us.

Moreover a mathematical problem should be difficult in order to entice us, yet not completely inaccessible, lest it mock at our efforts. It should be to us a guide

Reprinted from Bull. Amer. Math. Soc. 8 (July 1902), 437-479.

Originally published as Mathematische Probleme. Vortrag, gehalten auf dem internationalen Mathematike-Congress zu Paris 1900, Gött. Nachr. 1900, 253-297, Vandenhoeck \& Ruprecht, Göttingen. Translated for the Bulletin, with the author's permission, by Dr. Mary Winston Newson, 1902. 
post on the mazy paths to hidden truths, and ultimately a reminder of our pleasure in the successful solution.

The mathematicians of past centuries were accustomed to devote themselves to the solution of difficult particular problems with passionate zeal. They knew the value of difficult problems. I remind you only of the "problem of the line of quickest descent," proposed by John Bernoulli. Experience teaches, explains Bernoulli in the public announcement of this problem, that lofty minds are led to strive for the advance of science by nothing more than by laying before them difficult and at the same time useful problems, and he therefore hopes to earn the thanks of the mathematical world by following the example of men like Mersenne, Pascal, Fermat, Viviani and others and laying before the distinguished analysts of his time a problem by which, as a touchstone, they may test the value of their methods and measure their strength. The calculus of variations owes its origin to this problem of Bernoulli and to similar problems.

Fermat had asserted, as is well known, that the diophantine equation

$$
x^{n}+y^{n}=z^{n}
$$

( $x, y$ and $z$ integers) is unsolvable - except in certain self-evident cases. The attempt to prove this impossibility offers a striking example of the inspiring effect which such a very special and apparently unimportant problem may have upon science. For Kummer, incited by Fermat's problem, was led to the introduction of ideal numbers and to the discovery of the law of the unique decomposition of the numbers of a circular field into ideal prime factors - a law which to-day in its generalization to any algebraic field by Dedekind and Kronecker, stands at the center of the modern theory of numbers and whose significance extends far beyond the boundaries of number theory into the realm of algebra and the theory of functions.

To speak of a very different region of research, I remind you of the problem of three bodies. The fruitful methods and the far-reaching principles which Poincaré has brought into celestial mechanics and which are to-day recognized and applied in practical astronomy are due to the circumstance that he undertook to treat anew that difficult problem and to approach nearer a solution.

The two last mentioned problems - that of Fermat and the problem of the three bodies - seem to us almost like opposite poles - the former a free invention of pure reason, belonging to the region of abstract number theory, the latter forced upon us by astronomy and necessary to an understanding of the simplest fundamental phenomena of nature.

But it often happens also that the same special problem finds application in the most unlike branches of mathematical knowledge. So, for example, the problem of the shortest line plays a chief and historically important part in the foundations of geometry, in the theory of curved lines and surfaces, in mechanics and in the calculus of variations. And how convincingly has F. Klein, in his work on the icosahedron, pictured the significance which attaches to the problem of the regular polyhedra in elementary geometry, in group theory, in the theory of equations and in that of linear differential equations.

In order to throw light on the importance of certain problems, I may also refer to Weierstrass, who spoke of it as his happy fortune that he found at the outset of his scientific career a problem so important as Jacobi's problem of inversion on which to work. 
Having now recalled to mind the general importance of problems in mathematics, let us turn to the question from what sources this science derives its problems. Surely the first and oldest problems in every branch of mathematics spring from experience and are suggested by the world of external phenomena. Even the rules of calculation with integers must have been discovered in this fashion in a lower stage of human civilization, just as the child of to-day learns the application of these laws by empirical methods. The same is true of the first problems of geometry, the problems bequeathed us by antiquity, such as the duplication of the cube, the squaring of the circle; also the oldest problems in the theory of the solution of numerical equations, in the theory of curves and the differential and integral calculus, in the calculus of variations, the theory of Fourier series and the theory of potential - to say noting of the further abundance of problems properly belonging to mechanics, astronomy and physics.

But, in the further development of a branch of mathematics, the human mind, encouraged by the success of its solutions, becomes conscious of its independence. It evolves from itself alone, often without appreciable influence from without, by means of logical combination, generalization, specialization, by separating and collecting ideas in fortunate ways, new and fruitful problems, and appears then itself as the real questioner. Thus arose the problem of prime numbers and the other problems of number theory, Galois's theory of equations, the theory of algebraic invariants, the theory of abelian and automorphic functions; indeed almost all the nicer questions of modern arithmetic and function theory arise in this way.

In the meantime, while the creative power of pure reason is at work, the outer world again comes into play, forces upon us new questions from actual experience, opens up new branches of mathematics, and while we seek to conquer these new fields of knowledge for the realm of pure thought, we often find the answers to old unsolved problems and thus at the same time advance most successfully the old theories. And it seems to me that the numerous and surprising analogies and that apparently prearranged harmony which the mathematician so often perceives in the questions, methods and ideas of the various branches of his science, have their origin in this ever-recurring interplay between thought and experience.

It remains to discuss briefly what general requirements may be justly laid down for the solution of a mathematical problem. I should say first of all, this: that it shall be possible to establish the correctness of the solution by means of a finite number of steps based upon a finite number of hypotheses which are implied in the statement of the problem and which must always be exactly formulated. This requirement of logical deduction by means of a finite number of processes is simply the requirement of rigor in reasoning. Indeed the requirement of rigor, which has become proverbial in mathematics, corresponds to a universal philosophical necessity of our understanding; and, on the other hand, only by satisfying this requirement do the thought content and the suggestiveness of the problem attain their full effect. A new problem, especially when it comes from the world of outer experience, is like a young twig, which thrives and bears fruit only when it is grafted carefully and in accordance with strict horticultural rules upon the old stem, the established achievements of our mathematical science.

Besides it is an error to believe that rigor in the proof is the enemy of simplicity. On the contrary we find it confirmed by numerous examples that the rigorous method is at the same time the simpler and the more easily comprehended. The 
very effort for rigor forces us to find out simpler methods of proof. It also frequently leads the way to methods which are more capable of development than the old methods of less rigor. Thus the theory of algebraic curves experienced a considerable simplification and attained greater unity by means of the more rigorous function-theoretical methods and the consistent introduction of transcendental devices. Further, the proof that the power series permits the application of the four elementary arithmetical operations a well as the term by term differentiation and integration, and the recognition of the utility of the power series depending upon this proof contributed materially to the simplification of all analysis, particularly of the theory of elimination and the theory of differential equations, and also of the existence proofs demanded in those theories. But the most striking example for my statement is the calculus of variations. The treatment of the first and second variations of definite integrals required in part extremely complicated calculations, and the processes applied by the old mathematicians had not the needful rigor. Weierstrass showed us the way to a new and sure foundation of the calculus of variations. By the examples of the simple and double integral I will show briefly, at the close of my lecture, how this way leads at once to a surprising simplification of the calculus of variations. For in the demonstration of the necessary and sufficient criteria for the occurrence of a maximum and minimum, the calculation of the second variation and in part, indeed, the wearisome reasoning connected with the first variation may be completely dispensed with - to say nothing of the advance which is involved in the removal of the restriction to variations for which the differential coefficients of the function vary but slightly.

While insisting on rigor in the proof as a requirement for a perfect solution of a problem, I should like, on the other hand, to oppose the opinion that only the concepts of analysis, or even those of arithmetic alone, are susceptible of a fully rigorous treatment. This opinion, occasionally advocated by eminent men, I consider entirely erroneous. Such a one-sided interpretation of the requirement of rigor would soon lead to the ignoring of all concepts arising from geometry, mechanics and physics, to a stoppage of the flow of new material from the outside world, and finally, indeed, as a last consequence, to the rejection of the ideas of the continuum and of the irrational number. But what an important nerve, vital to mathematical science, would be cut by the extirpation of geometry and mathematical physics! On the contrary I think that wherever, from the side of the theory of knowledge or in geometry, or from the theories of natural or physical science, mathematical ideas come up, the problem arises for mathematical science to investigate the principles underlying these ideas and so to establish them upon a simple and complete system of axioms, that the exactness of the new ideas and their applicability to deduction shall be in no respect inferior to those of the old arithmetical concepts.

To new concepts correspond, necessarily, new signs. These we choose in such a way that they remind us of the phenomena which were the occasion for the formation of the new concepts. So the geometrical figures are signs or mnemonic symbols of space intuition and are used as such by all mathematicians. Who does not always use along with the double inequality $a>b>c$ the picture of three points following one another on a straight line as the geometrical picture of the idea "between"? Who does not make use of drawings of segments and rectangles enclosed in one another, when it is required to prove with perfect rigor a difficult theorem on the continuity of functions or the existence of points of condensation? Who could dispense with the figure of the triangle, the circle with its center, or with the cross 
of three perpendicular axes? Or who would give up the representation of the vector field, or the picture of a family of curves or surfaces with its envelope which plays so important a part in differential geometry, in the theory of differential equations, in the foundation of the calculus of variations and in other purely mathematical sciences?

The arithmetical symbols are written diagrams and the geometrical figures are graphic formulas; and no mathematician could spare these graphic formulas, any more than in calculation the insertion and removal of parentheses or the use of other analytical signs.

The use of geometrical signs as a means of strict proof presupposes the exact knowledge and complete mastery of the axioms which underlie those figures; and in order that these geometrical figures may be incorporated in the general treasure of mathematical signs, there is necessary a rigorous axiomatic investigation of their conceptual content. Just as in adding two numbers, one must place the digits under each other in the right order, so that only the rules of calculation, i.e., the axioms of arithmetic, determine the correct use of the digits, so the use of geometrical signs is determined by the axioms of geometrical concepts and their combinations.

The agreement between geometrical and arithmetical thought is shown also in that we do not habitually follow the chain of reasoning back to the axioms in arithmetical, any more than in geometrical discussions. On the contrary we apply, especially in first attacking a problem, a rapid, unconscious, not absolutely sure combination, trusting to a certain arithmetical feeling for the behavior of the arithmetical symbols, which we could dispense with as little in arithmetic as with the geometrical imagination in geometry. As an example of an arithmetical theory operating rigorously with geometrical ideas and signs, I may mention Minkowski's work, Die Geometrie der Zahlen.1

Some remarks upon the difficulties which mathematical problems may offer, and the means of surmounting them, may be in place here.

If we do not succeed in solving a mathematical problem, the reason frequently consists in our failure to recognize the more general standpoint from which the problem before us appears only as a single link in a chain of related problems. After finding this standpoint, not only is this problem frequently more accessible to our investigation, but at the same time we come into possession of a method which is applicable also to related problems. The introduction of complex paths of integration by Cauchy and of the notion of the IDEALS in number theory by Kummer may serve as examples. This way for finding general methods is certainly the most practicable and the most certain; for he who seeks for methods without having a definite problem in mind seeks for the most part in vain.

In dealing with mathematical problems, specialization plays, as I believe, a still more important part than generalization. Perhaps in most cases where we seek in vain the answer to a question, the cause of the failure lies in the fact that problems simpler and easier than the one in hand have been either not at all or incompletely solved. All depends, then, on finding out these easier problems, and on solving them by means of devices as perfect as possible and of concepts capable of generalization. This rule is one of the most important leers for overcoming mathematical difficulties and it seems to me that it is used almost always, though perhaps unconsciously.

\footnotetext{
${ }^{1}$ Leipzig, 1896.
} 
Occasionally it happens that we seek the solution under insufficient hypotheses or in an incorrect sense, and for this reason do not succeed. The problem then arises: to show the impossibility of the solution under the given hypotheses, or in the sense contemplated. Such proofs of impossibility were effected by the ancients, for instance when they showed that the ratio of the hypotenuse to the side of an isosceles right triangle is irrational. In later mathematics, the question as to the impossibility of certain solutions plays a preëminent part, and we perceive in this way that old and difficult problems, such as the proof of the axiom of parallels, the squaring of the circle, or the solution of equations of the fifth degree by radicals have finally found fully satisfactory and rigorous solutions, although in another sense than that originally intended. It is probably this important fact along with other philosophical reasons that gives rise to the conviction (which every mathematician shares, but which no one has as yet supported by a proof) that every definite mathematical problem must necessarily be susceptible of an exact settlement, either in the form of an actual answer to the question asked, or by the proof of the impossibility of its solution and therewith the necessary failure of all attempts. Take any definite unsolved problem, such as the question as to the irrationality of the Euler-Mascheroni constant $C$, or the existence of an infinite number of prime numbers of the form $2^{n}+1$. However unapproachable these problems may seem to us and however helpless we stand before them, we have, nevertheless, the firm conviction that their solution must follow by a finite number of purely logical processes.

Is this axiom of the solvability of every problem a peculiarity characteristic of mathematical thought alone, or is it possibly a general law inherent in the nature of the mind, that all questions which it asks must be answerable? For in other sciences also one meets old problems which have been settled in a manner most satisfactory and most useful to science by the proof of their impossibility. I instance the problem of perpetual motion. After seeking in vain for the construction of a perpetual motion machine, the relations were investigated which must subsist between the forces of nature if such a machine is to be impossible 2 and this inverted question led to the discovery of the law of the conservation of energy, which, again, explained the impossibility of perpetual motion in the sense originally intended.

This conviction of the solvability of every mathematical problem is a powerful incentive to the worker. We hear within us the perpetual call: There is the problem. Seek its solution. You can find it by pure reason, for in mathematics there is no ignorabimus.

The supply of problems in mathematics is inexhaustible, and as soon as one problem is solved numerous others come forth in its place. Permit me in the following, tentatively as it were, to mention particular definite problems, drawn from various branches of mathematics, from the discussion of which an advancement of science may be expected.

Let us look at the principles of analysis and geometry. The most suggestive and notable achievements of the last century in this field are, as it seems to me, the arithmetical formulation of the concept of the continuum in the works of Cauchy, Bolzano and Cantor, and the discovery of non-euclidean geometry by Gauss, Bolyai,

\footnotetext{
${ }^{2}$ See Helmholtz, "Ueber die Wechselwirkung der Naturkräefte und die darauf bezüglichen neuesten Ermittelungen der Physik"; Vortrag, gehalten in Königsberg, 1854.
} 
and Lobachevsky. I therefore first direct your attention to some problems belonging to these fields.

\section{Cantor's Problem of the Cardinal number of the Continuum}

Two systems, i.e., two assemblages of ordinary real numbers or points, are said to be (according to Cantor) equivalent or of equal cardinal number, if they can be brought into a relation to one another such that to every number of the one assemblage corresponds one and only one definite number of the other. The investigations of Cantor on such assemblages of points suggest a very plausible theorem, which nevertheless, in spite of the most strenuous efforts, no one has succeeded in proving. This is the theorem:

Every system of infinitely many real numbers, i.e., every assemblage of numbers (or points), is either equivalent to the assemblage of natural integers, $1,2,3, \ldots$ or to the assemblage of all real numbers and therefore to the continuum, that is, to the points of a line; as regards equivalence there are, therefore, only two assemblages of numbers, the countable assemblage and the continuum.

From this theorem it would follow at once that the continuum has the next cardinal number beyond that of the countable assemblage; the proof of this theorem would, therefore, form a new bridge between the countable assemblage and the continuum.

Let me mention another very remarkable statement of Cantor's which stands in the closest connection with the theorem mentioned and which, perhaps, offers the key to its proof. Any system of real numbers is said to be ordered, if for every two numbers of the system it is determined which one is the earlier and which the later, and if at the same time this determination is of such a kind that, if $a$ is before $b$ and $b$ is before $c$, then $a$ always comes before $c$. The natural arrangement of numbers of a system is defined to be that in which the smaller precedes the larger. But there are, as is easily seen, infinitely many other ways in which the numbers of a system may be arranged.

If we think of a definite arrangement of numbers and select from them a particular system of these numbers, a so-called partial system or assemblage, this partial system will also prove to be ordered. Now Cantor considers a particular kind of ordered assemblage which he designates as a well ordered assemblage and which is characterized in this way, that not only in the assemblage itself but also in every partial assemblage there exists a first number. The system of integers $1,2,3, \ldots$ in their natural order is evidently a well ordered assemblage. On the other hand the system of all real numbers, i.e., the continuum in its natural order, is evidently not well ordered. For, if we think of the points of a segment of a straight line, with its initial point excluded, as our partial assemblage, it will have no first element.

The question now arises whether the totality of all numbers may not be arranged in another manner so that every partial assemblage may have a first element, i.e., whether the continuum cannot be considered as a well ordered assemblage - a question which Cantor thinks must be answered in the affirmative. It appears to me most desirable to obtain a direct proof of this remarkable statement of Cantor's, perhaps by actually giving an arrangement of numbers such that in every partial system a first number can be pointed out. 


\section{THE COMPATIBILITY OF THE ARITHMETICAL AXIOMS}

When we are engaged in investigating the foundations of a science, we must set up a system of axioms which contains an exact and complete description of the relations subsisting between the elementary ideas of that science. The axioms so set up are at the same time the definitions of those elementary ideas; and no statement within the realm of the science whose foundation we are testing is held to be correct unless it can be derived from those axioms by means of a finite number of logical steps. Upon closer consideration the question arises: Whether, in any way, certain statements of single axioms depend upon one another, and whether the axioms may not therefore contain certain parts in common, which must be isolated if one wishes to arrive at a system of axioms that shall be altogether independent of one another.

But above all I wish to designate the following as the most important among the numerous questions which can be asked with regard to the axioms: To prove that they are not contradictory, that is, that a finite number of logical steps based upon them can never lead to contradictory results.

In geometry, the proof of the compatibility of the axioms can be effected by constructing a suitable field of numbers, such that analogous relations between the numbers of this field correspond to the geometrical axioms. Any contradiction in the deductions from the geometrical axioms must thereupon be recognizable in the arithmetic of this field of numbers. In this way the desired proof for the compatibility of the geometrical axioms is made to depend upon the theorem of the compatibility of the arithmetical axioms.

On the other hand a direct method is needed for the proof of the compatibility of the arithmetical axioms. The axioms of arithmetic are essentially nothing else than the known rules of calculation, with the addition of the axiom of continuity. I recently collected them 3 and in so doing replaced the axiom of continuity by two simpler axioms, namely, the well-known axiom of Archimedes, and a new axiom essentially as follows: that numbers form a system of things which is capable of no further extension, as long as all the other axioms hold (axiom of completeness). I am convinced that it must be possible to find a direct proof for the compatibility of the arithmetical axioms, by means of a careful study and suitable modification of the known methods of reasoning in the theory of irrational numbers.

To show the significance of the problem from another point of view, I add the following observation: If contradictory attributes be assigned to a concept, I say, that mathematically the concept does not exist. So, for example, a real number whose square is -1 does not exist mathematically. But if it can be proved that the attributes assigned to the concept can never lead to a contradiction by the application of a finite number of logical processes, I say that the mathematical existence of the concept (for example, of a number or a function which satisfies certain conditions) is thereby proved. In the case before us, where we are concerned with the axioms of real numbers in arithmetic, the proof of the compatibility of the axioms is at the same time the proof of the mathematical existence of the complete system of real numbers or of the continuum. Indeed, when the proof for the compatibility of the axioms shall be fully accomplished, the doubts which have been expressed occasionally as to the existence of the complete system of real numbers will become totally groundless. The totality of real numbers, i.e., the continuum according to the point of view just indicated, is not the totality of

\footnotetext{
${ }^{3}$ Jahresbericht der Deutschen Mathematiker-Vereinigung, vol. 8 (1900), p. 180.
} 
all possible series in decimal fractions, or of all possible laws according to which the elements of a fundamental sequence may proceed. It is rather a system of things whose mutual relations are governed by the axioms set up and for which all propositions, and only those, are true which can be derived from the axioms by a finite number of logical processes. In my opinion, the concept of the continuum is strictly logically tenable in this sense only. It seems to me, indeed, that this corresponds best also to what experience and intuition tell us. The concept of the continuum or even that of the system of all functions exists, then, in exactly the same sense as the system of integral, rational numbers, for example, or as Cantor's higher classes of numbers and cardinal numbers. For I am convinced that the existence of the latter, just as that of the continuum, can be proved in the sense I have described; unlike the system of all cardinal numbers or of all Cantor's alephs, for which, as may be shown, a system of axioms, compatible in my sense, cannot be set up. Either of these systems is, therefore, according to my terminology, mathematically non-existent.

From the field of the foundations of geometry I should like to mention the following problem:

\section{The EQUAlity of THE VOlumes OF TWO TETRAHEDRA OF EQUAL BASES AND EQUAL ALTITUDES}

In two letters to Gerling, Gaus 4 expresses his regret that certain theorems of solid geometry depend upon the method of exhaustion, i.e., in modern phraseology, upon the axiom of continuity (or upon the axiom of Archimedes). Gauss mentions in particular the theorem of Euclid, that triangular pyramids of equal altitudes are to each other as their bases. Now the analogous problem in the plane has been solved 5 Gerling also succeeded in proving the equality of volume of symmetrical polyhedra by dividing them into congruent parts. Nevertheless, it seems to me probable that a general proof of this kind for the theorem of Euclid just mentioned is impossible, and it should be our task to give a rigorous proof of its impossibility. This would be obtained, as soon as we succeeded in specifying two tetrahedra of equal bases and equal altitudes which can in no way be split up into congruent tetrahedra, and which cannot be combined with congruent tetrahedra to form two polyhedra which themselves could be split up into congruent tetrahedra 6

\section{Problem of the Straight line as the SHORTEST DISTANCE BETWEEN TWO POINTS}

Another problem relating to the foundations of geometry is this: If from among the axioms necessary to establish ordinary euclidean geometry, we exclude the axiom of parallels, or assume it as not satisfied, but retain all other axioms, we obtain, as is well known, the geometry of Lobachevsky (hyperbolic geometry). We may therefore say that this is a geometry standing next to euclidean geometry. If we require further that that axiom be not satisfied whereby, of three points of a straight line, one and only one lies between the other two, we obtain Riemann's (elliptic)

\footnotetext{
${ }^{4}$ Werke, vol. 8, pp. 241 and 244.

${ }^{5}$ Cf., beside earlier literature, Hilbert, Grundlagen der Geometrie, Leipzig, 1899, ch. 4. [Translation by Townsend, Chicago, 1902.]

${ }^{6}$ Since this was written Herr Dehn has succeeded in proving this impossibility. See his note: "Ueber raumgleiche Polyeder", in Nachrichten d. K. Geselsch. d. Wiss. zu Göttingen, 1900, and a paper soon to appear in the Math. Annalen [vol. 55, pp. 465-478].
} 
geometry, so that this geometry appears to be the next after Lobachevsky's. If we wish to carry out a similar investigation with respect to the axiom of Archimedes, we must look upon this as not satisfied, and we arrive thereby at the non-archimedean geometries which have been investigated by Veronese and myself. The more general question now arises: Whether from other suggestive standpoints geometries may not be devised which, with equal right, stand next to euclidean geometry. Here I should like to direct your attention to a theorem which has, indeed, been employed by many authors as a definition of a straight line, viz., that the straight line is the shortest distance between two points. The essential content of this statement reduces to the theorem of Euclid that in a triangle the sum of two sides is always greater than the third side - a theorem which, as is easily seen, deals solely with elementary concepts, i.e., with such as are derived directly from the axioms, and is therefore more accessible to logical investigation. Euclid proved this theorem, with the help of the theorem of the exterior angle, on the basis of the congruence theorems. Now it is readily shown that this theorem of Euclid cannot be proved solely on the basis of those congruence theorems which relate to the application of segments and angles, but that one of the theorems on the congruence of triangles is necessary. We are asking, then, for a geometry in which all the axioms of ordinary euclidean geometry hold, and in particular all the congruence axioms except the one of the congruence of triangles (or all except the theorem of the equality of the base angles in the isosceles triangle), and in which, besides, the proposition that in every triangle the sum of two sides is greater than the third is assumed as a particular axiom.

One finds that such a geometry really exists and is no other than that which Minkowski constructed in his book, Geometrie der Zahlen 7 and made the basis of his arithmetical investigations. Minkowski's is therefore also a geometry standing next to the ordinary euclidean geometry; it is essentially characterized by the following stipulations:

1. The points which are at equal distances from a fixed point $O$ lie on a convex closed surface of the ordinary euclidean space with $O$ as a center.

2. Two segments are said to be equal when one can be carried into the other by a translation of the ordinary euclidean space.

In Minkowski's geometry the axiom of parallels also holds. By studying the theorem of the straight line as the shortest distance between two points, I arrived 8 at a geometry in which the parallel axiom does not hold, while all other axioms of Minkowski's geometry are satisfied. The theorem of the straight line as the shortest distance between two points and the essentially equivalent theorem of Euclid about the sides of a triangle, play an important part not only in number theory but also in the theory of surfaces and in the calculus of variations. For this reason, and because I believe that the thorough investigation of the conditions for the validity of this theorem will throw a new light upon the idea of distance, as well as upon other elementary ideas, e.g., upon the idea of the plane, and the possibility of its definition by means of the idea of the straight line, the construction and systematic treatment of the geometries here possible seem to me desirable.

\footnotetext{
${ }^{7}$ Leipzig, 1896.

${ }^{8}$ Math. Annalen, vol. 46, p. 91.
} 


\section{LIE'S CONCEPT OF A CONTINUOUS GROUP OF TRANSFORMATIONS WITHOUT THE ASSUMPTION OF THE DIFFERENTIABILITY OF THE FUNCTIONS DEFINING THE GROUP}

It is well known that Lie, with the aid of the concept of continuous groups of transformations, has set up a system of geometrical axioms and, from the standpoint of his theory of groups, has proved that this system of axioms suffices for geometry. But since Lie assumes, in the very foundation of his theory, that the functions defining his group can be differentiated, it remains undecided in Lie's development, whether the assumption of the differentiability in connection with the question as to the axioms of geometry is actually unavoidable, or whether it may not appear rather as a consequence of the group concept and the other geometrical axioms. This consideration, as well as certain other problems in connection with the arithmetical axioms, brings before us the more general question: How far Lie's concept of continuous groups of transformations is approachable in our investigations without the assumption of the differentiability of the functions.

Lie defines a finite continuous group of transformations as a system of transformations

$$
x_{i}^{\prime}=f_{i}\left(x_{1}, \ldots, x_{n} ; a_{1}, \ldots, a_{r}\right) \quad(i=1, \ldots, n)
$$

having the property that any two arbitrarily chosen transformations of the system, as

$$
\begin{aligned}
x_{i}^{\prime} & =f_{i}\left(x_{1}, \ldots, x_{n} ; a_{1}, \ldots, a_{r}\right), \\
x_{i}^{\prime \prime} & =f_{i}\left(x_{1}^{\prime}, \ldots, x_{n}^{\prime} ; b_{1}, \ldots, b_{r}\right),
\end{aligned}
$$

applied successively result in a transformation which also belongs to the system, and which is therefore expressible in the form

$$
x_{i}^{\prime \prime}=f_{i}\left\{f_{1}(x, a), \ldots, f_{n}(x, a) ; b_{1}, \ldots, b_{r}\right\}=f_{i}\left(x_{1}, \ldots, x_{n} ; c_{1}, \ldots, c_{r}\right),
$$

where $c_{1}, \ldots, c_{r}$ are certain functions of $a_{1}, \ldots, a_{r}$ and $b_{1}, \ldots, b_{r}$. The group property thus finds its full expression in a system of functional equations and of itself imposes no additional restrictions upon the functions $f_{1}, \ldots, f_{n} ; c_{1}, \ldots, c_{r}$. Yet Lie's further treatment of these functional equations, viz., the derivation of the well-known fundamental differential equations, assumes necessarily the continuity and differentiability of the functions defining the group.

As regards continuity: this postulate will certainly be retained for the presentif only with a view to the geometrical and arithmetical applications, in which the continuity of the functions in question appears as a consequence of the axiom of continuity. On the other hand the differentiability of the functions defining the group contains a postulate which, in the geometrical axioms, can be expressed only in a rather forced and complicated manner. Hence there arises the question whether, through the introduction of suitable new variables and parameters, the group can always be transformed into one whose defining functions are differentiable; or whether, at least with the help of certain simple assumptions, a transformation is possible into groups admitting Lie's methods. A reduction to analytic groups is, according to a theorem announced by Li\&9 but first proved by

${ }^{9}$ Lie-Engel, Theorie der Transformationsgruppen, vol. 3, Leipzig, 1893, §§82, 144. 
Schur 10 always possible when the group is transitive and the existence of the first and certain second derivatives of the functions defining the group is assumed.

For infinite groups the investigation of the corresponding question is, I believe, also of interest. Moreover we are thus led to the wide and interesting field of functional equations which have been heretofore investigated usually only under the assumption of the differentiability of the functions involved. In particular the functional equations treated by Abe 11 with so much ingenuity, the difference equations, and other equations occurring in the literature of mathematics, do not directly involve anything which necessitates the requirement of the differentiability of the accompanying functions. In the search for certain existence proofs in the calculus of variations I came directly upon the problem: To prove the differentiability of the function under consideration from the existence of a difference equation. In all these cases, then, the problem arises: In how far are the assertions which we can make in the case of differentiable functions true under proper modifications without this assumption?

It may be further remarked that H. Minkowski in his above-mentioned Geometrie der Zahlen starts with the functional equation

$$
f\left(x_{1}+y_{1}, \ldots, x_{n}+y_{n}\right) \leq f\left(x_{1}, \ldots, x_{n}\right)+f\left(y_{1}, \ldots, y_{n}\right)
$$

and from this actually succeeds in proving the existence of certain differential quotients for the function in question.

On the other hand I wish to emphasize the fact that there certainly exist analytical functional equations whose sole solutions are non-differentiable functions. For example a uniform continuous non-differentiable function $\varphi(x)$ can be constructed which represents the only solution of the two functional equations

$$
\varphi(x+\alpha)-\varphi(x)=f(x), \quad \varphi(x+\beta)-\varphi(x)=0,
$$

where $\alpha$ and $\beta$ are two real numbers, and $f(x)$ denotes, for all the real values of $x$, a regular analytic uniform function. Such functions are obtained in the simplest manner by means of trigonometrical series by a process similar to that used by Borel (according to a recent announcement of Picard) 12 for the construction of a doubly periodic, non-analytic solution of a certain analytic partial differential equation.

\section{Mathematical tREATMENT OF THE AXIOMS OF PHYSiCS}

The investigations on the foundations of geometry suggest the problem: To treat in the same manner, by means of axioms, those physical sciences in which mathematics plays an important part; in the first rank are the theory of probabilities and mechanics.

As to the axioms of the theory of probabilities 13 it seems to me desirable that their logical investigation should be accompanied by a rigorous and satisfactory development of the method of mean values in mathematical physics, and in particular in the kinetic theory of gases.

\footnotetext{
10 "Ueber den analytischen Charakter der eine endliche Kontinuierliche Transformationsgruppen darstellenden Funktionen", Math. Annalen, vol. 41.

${ }^{11}$ Werke, vol. 1, pp. 1, 61, 389.

12 "Quelques théories fondamentales dans l'analyse mathématique", Conférences faites à Clark University, Revue générale des Sciences, 1900, p. 22.

${ }^{13}$ Cf. Bohlmann, "Ueber Versicherungsmathematik", from the collection: Klein and Riecke, Ueber angewandte Mathematik und Physik, Leipzig, 1900.
} 
Important investigations by physicists on the foundations of mechanics are at hand; I refer to the writings of Mach.14 Hertz 15 Boltzmann 16 and Volkmann 17 It is therefore very desirable that the discussion of the foundations of mechanics be taken up by mathematicians also. Thus Boltzmann's work on the principles of mechanics suggests the problem of developing mathematically the limiting processes, there merely indicated, which lead from the atomistic view to the laws of motion of continua. Conversely one might try to derive the laws of the motion of rigid bodies by a limiting process from a system of axioms depending upon the idea of continuously varying conditions of a material filling all space continuously, these conditions being defined by parameters. For the question as to the equivalence of different systems of axioms is always of great theoretical interest.

If geometry is to serve as a model for the treatment of physical axioms, we shall try first by a small number of axioms to include as large a class as possible of physical phenomena, and then by adjoining new axioms to arrive gradually at the more special theories. At the same time Lie's principle of subdivision can perhaps be derived from profound theory of infinite transformation groups. The mathematician will have also to take account not only of those theories coming near to reality, but also, as in geometry, of all logically possible theories. He must be always alert to obtain a complete survey of all conclusions derivable from the system of axioms assumed.

Further, the mathematician has the duty to test exactly in each instance whether the new axioms are compatible with the previous ones. The physicist, as his theories develop, often finds himself forced by the results of his experiments to make new hypotheses, while he depends, with respect to the compatibility of the new hypotheses with the old axioms, solely upon these experiments or upon a certain physical intuition, a practice which in the rigorously logical building up of a theory is not admissible. The desired proof of the compatibility of all assumptions seems to me also of importance, because the effort to obtain each proof always forces us most effectually to an exact formulation of the axioms.

So far we have considered only questions concerning the foundations of the mathematical sciences. Indeed, the study of the foundations of a science is always particularly attractive, and the testing of these foundations will always be among the foremost problems of the investigator. Weierstrass once said, "The final object always to be kept in mind is to arrive at a correct understanding of the foundations of the science....But to make any progress in the sciences the study of particular problems is, of course, indispensable." In fact, a thorough understanding of its special theories is necessary to the successful treatment of the foundations of the science. Only that architect is in the position to lay a sure foundation for a structure who knows its purpose thoroughly and in detail. So we turn now to the special problems of the separate branches of mathematics and consider first arithmetic and algebra.

\footnotetext{
${ }^{14}$ Die Mechanik in ihrer Entwickelung, Leipzig, 4th edition, 1901.

${ }^{15}$ Die Prinzipien der Mechanik, Leipzig, 1894.

${ }^{16}$ Vorlesungen über die Principe der Mechanik, Leipzig, 1897.

${ }^{17}$ Einführung in das Studium der theoretischen Physik, Leipzig, 1900.
} 


\section{IRRATIONALITY AND TRANSCENDENCE OF CERTAIN NUMBERS}

Hermite's arithmetical theorems on the exponential function and their extension by Lindemann are certain of the admiration of all generations of mathematicians. Thus the task at once presents itself to penetrate further along the path here entered, as A. Hurwitz has already done in two interesting papers 18 "Ueber arithmetische Eigenschaften gewisser transzendenter Funktionen". I should like, therefore, to sketch a class of problems which, in my opinion, should be attacked as here next in order. That certain special transcendental functions, important in analysis, take algebraic values for certain algebraic arguments, seems to us particularly remarkable and worthy of thorough investigation. Indeed, we expect transcendental functions to assume, in general, transcendental values for even algebraic arguments; and, although it is well known that there exist integral transcendental functions which even have rational values for all algebraic arguments, we shall still consider it highly probable that the exponential functions $e^{i \pi z}$, for example, which evidently has algebraic values for all rational arguments $z$, will on the other hand always take transcendental values for irrational algebraic values of the argument $z$. We can also give this statement a geometrical form, as follows:

If, in an isosceles triangle, the ratio of the base angle to the angle at the vertex be algebraic but not rational, the ratio between base and side is always transcendental.

In spite of the simplicity of this statement and of its similarity to the problems solved by Hermite and Lindemann, I consider the proof of this theorem very difficult; as also the proof that

The expression $\alpha^{\beta}$, for an algebraic base $\alpha$ and an irrational algebraic exponent $\beta$, e.g., the number $2^{\sqrt{2}}$ or $e^{\pi}=i^{-2 i}$, always represents a transcendental or at least an irrational number.

It is certain that the solution of these and similar problems must lead us to entirely new methods and to a new insight into the nature of special irrational and transcendental numbers.

\section{Problems of PRIME Numbers}

Essential progress in the theory of the distribution of prime numbers has lately been made by Hadamard, de la Vallée-Poussin, Von Mangoldt and others. For the complete solution, however, of the problems set us by Riemann's paper "Ueber die Anzahl der Primzahlen unter einer gegebenen Grösse", it still remains to prove the correctness of an exceedingly important statement of Riemann, viz., that the zero points of the function $\zeta(s)$ defined by the series

$$
\zeta(s)=1+\frac{1}{2^{s}}+\frac{1}{3^{s}}+\frac{1}{4^{s}}+\cdots
$$

all have the real part $\frac{1}{2}$, except the well-known negative integral real zeros. As soon as this proof has been successfully established, the next problem would consist in testing more exactly Riemann's infinite series for the number of primes below a given number and, especially, to decide whether the difference between the number of primes below a number $x$ and the integral logarithm of $x$ does in fact become infinite of an order not greater than $\frac{1}{2}$ in $x 19$ Further, we should determine whether

\footnotetext{
${ }^{18}$ Math. Annalen, vols. 22, 32 (1883, 1888).

${ }^{19} \mathrm{Cf}$. an article by H. von Koch, which is soon to appear in the Math. Annalen [Vol. 55, p. 441].
} 
the occasional condensation of prime numbers which has been noticed in counting primes is really due to those terms of Riemann's formula which depend upon the first complex zeros of the function $\zeta(s)$.

After an exhaustive discussion of Riemann's prime number formula, perhaps we may sometime be in a position to attempt the rigorous solution of Goldbach's problem 20 viz., whether every integer is expressible as the sum of two positive prime numbers; and further to attack the well-known question, whether there are an infinite number of pairs of prime numbers with the difference 2, or even the more general problem, whether the linear diophantine equation

$$
a x+b y+c=0
$$

(with given integral coefficients each prime to the others) is always solvable in prime numbers $x$ and $y$.

But the following problem seems to me of no less interest and perhaps of still wider range: To apply the results obtained for the distribution of rational prime numbers to the theory of the distribution of ideal primes in a given number-field $k$-a problem which looks toward the study of the function $\zeta_{k}(s)$ belonging to the field and defined by the series

$$
\zeta_{k}(s)=\sum \frac{1}{n(j)^{s}}
$$

where the sum extends over all ideals $j$ of the given realm $k$, and $n(j)$ denotes the norm of the ideal $j$.

I may mention three more special problems in number theory: one on the laws of reciprocity, one on diophantine equations, and a third from the realm of quadratic forms.

\section{Proof of the most General LAW of Reciprocity IN ANY NUMBER FIELD}

For any field of numbers the law of reciprocity is to be proved for the residues of the $l$ th power, when $l$ denotes an odd prime, and further when $l$ is a power of 2 or a power of an odd prime.

The law, as well as the means essential to its proof, will, I believe, result by suitably generalizing the theory of the field of the $l$ th roots of unity 21 developed by me, and my theory of relative quadratic fields 22

\section{Determination of the solvability of A Diophantine EQUATion}

Given a diophantine equation with any number of unknown quantities and with rational integral numerical coefficients: To devise a process according to which it can be determined by a finite number of operations whether the equation is solvable in rational integers.

\footnotetext{
${ }^{20}$ Cf. P. Stäckel: "Über Goldbach's empirisches Theorem", Nachrichten d. K. Ges. d. Wiss. zu Göttingen, 1896, and Landau, ibid., 1900.

${ }^{21}$ Jahresber. d. Deutschen Math.-Vereinigung, "Ueber die Theorie der algebraischen Zahlkörper", vol. 4 (1897), Part V.

${ }^{22}$ Math. Annalen, vol. 51 and Nachrichten d. K. Ges. d. Wiss. zu Göttingen, 1898.
} 


\section{QuAdRATiC FORMS With ANy ALGEBRAiC NUMERICAL COEFFICIENTS}

Our present knowledge of the theory of quadratic number field 23 puts us in a position to attack successfully the theory of quadratic forms with any number of variables and with any algebraic numerical coefficients. This leads in particular to the interesting problem: to solve a given quadratic equation with algebraic numerical coefficients in any number of variables by integral or fractional numbers belonging to the algebraic realm of rationality determined by the coefficients.

The following important problem may form a transition to algebra and the theory of functions:

\section{EXTENSION OF KRONECKER'S THEOREM ON ABELIAN FIELDS TO ANY ALGEBRAIC REALM OF RATIONALITY}

The theorem that every abelian number field arises from the realm of rational numbers by the composition of fields of roots of unity is due to Kronecker. This fundamental theorem in the theory of integral equations contains two statements, namely:

First. It answers the question as to the number and existence of those equations which have a given degree, a given abelian group and a given discriminant with respect to the realm of rational numbers.

Second. It states that the roots of such equations form a realm of algebraic numbers which coincides with the realm obtained by assigning to the argument $z$ in the exponential function $e^{i \pi z}$ all rational numerical values in succession.

The first statement is concerned with the question of the determination of certain algebraic numbers by their groups and their branching. This question corresponds, therefore, to the known problem of the determination of algebraic functions corresponding to given Riemann surfaces. The second statement furnishes the required numbers by transcendental means, namely, by the exponential function $e^{i \pi z}$.

Since the realm of the imaginary quadratic number fields is the simplest after the realm of rational numbers, the problem arises, to extend Kronecker's theorem to this case. Kronecker himself has made the assertion that the abelian equations in the realm of a quadratic field are given by the equations of transformation of elliptic functions with singular moduli, so that the elliptic function assumes here the same rôle as the exponential function in the former case. The proof of Kronecker's conjecture has not yet been furnished; but I believe that it must be obtainable without very great difficulty on the basis of the theory of complex multiplication developed by H. Weber ${ }^{24}$ with the help of the purely arithmetical theorems on class fields which I have established.

Finally, the extension of Kronecker's theorem to the case that, in place of the realm of rational numbers or of the imaginary quadratic field, any algebraic field

\footnotetext{
${ }^{23}$ Hilbert, "Ueber den Dirichlet'schen biquadratischen Zahlenkörper", Math. Annalen, vol. 45; "Ueber die Theorie der relativquadratischen Zahlkörper", Jahresber. d. Deutschen MathematikerVereinigung, 1897, and Math. Annalen, vol. 51; "Ueber die Theorie der relativ-Abelschen Körper", Nachrichten d. K. Ges. d. Wiss. zu Göttingen, 1898; Grundlagen der Geometrie, Leipzig, 1899, Chap. VIII, $\S 83$ [Translation by Townsend, Chicago, 1902]. Cf. also the dissertation of G. Rückle, Göttingen, 1901.

${ }^{24}$ Elliptische Functionen und algebraische Zahlen, Braunschweig, 1891.
} 
whatever is laid down as realm of rationality, seems to me of the greatest importance. I regard this problem as one of the most profound and far-reaching in the theory of numbers and of functions.

The problem is found to be accessible from many standpoints. I regard as the most important key to the arithmetical part of this problem the general law of reciprocity for residues of $l$ th powers within any given number field.

As to the function-theoretical part of the problem, the investigator in this attractive region will be guided by the remarkable analogies which are noticeable between the theory of algebraic functions of one variable and the theory of algebraic numbers. Hense 25 has proposed and investigated the analogue in the theory of algebraic numbers to the development in power series of an algebraic function; and Landsberg 26 has treated the analogue of the Riemann-Roch theorem. The analogy between the deficiency of a Riemann surface and that of the class number of a field of numbers is also evident. Consider a Riemann surface of deficiency $p=1$ (to touch on the simplest case only) and on the other hand a number field of class $h=2$. To the proof of the existence of an integral everywhere finite on the Riemann surface, corresponds the proof of the existence of an integer $\alpha$ in the number field such that the number $\sqrt{\alpha}$ represents a quadratic field, relatively unbranched with respect to the fundamental field. In the theory of algebraic functions, the method of boundary values (Randwerthaufgabe) serves, as is well known, for the proof of Riemann's existence theorem. In the theory of number fields also, the proof of the existence of just this number $\alpha$ offers the greatest difficulty. This proof succeeds with indispensable assistance from the theorem that in the number field there are always prime ideals corresponding to given residual properties. This latter fact is therefore the analogue in number theory to the problem of boundary values.

The equation of Abel's theorem in the theory of algebraic functions expresses, as is well known, the necessary and sufficient condition that the points in question on the Riemann surface are the zero points of an algebraic function belonging to the surface. The exact analogue of Abel's theorem, in the theory of the number field of class $h=2$, is the equation of the law of quadratic reciprocity 27

$$
\left(\frac{\alpha}{j}\right)=+1
$$

which declares that the ideal $j$ is then and only then a principal ideal of the number field when the quadratic residue of the number $\alpha$ with respect to the ideal $j$ is positive.

It will be seen that in the problem just sketched the three fundamental branches of mathematics, number theory, algebra and function theory, come into closest touch with one another, and I am certain that the theory of analytical functions of several variables in particular would be notably enriched if one should succeed in finding and discussing those functions which play the part for any algebraic number field corresponding to that of the exponential function in the field of rational numbers and of the elliptic modular functions in the imaginary quadratic number field.

\footnotetext{
${ }^{25}$ Jahresber. $d$. Deutschen Math.-Vereinigung, vol. 6, and an article soon to appear in the Math. Annalen [Vol. 55, p. 301]: "Ueber die Entwickelung der algebraischen Zahlen in Potenzreihen".

${ }^{26}$ Math. Annalen, vol. 50 (1898).

${ }^{27}$ Cf. Hilbert, "Ueber die Theorie der relativ-Abelschen Zahlkörper", Gött. Nachrichten, 1898.
} 
Passing to algebra, I shall mention a problem from the theory of equations and one to which the theory of algebraic invariants has led me.

\author{
13. IMPOSSIBILITY OF THE SOLUTION OF THE \\ GENERAL EQUATION OF THE 7TH DEGREE BY MEANS \\ OF FUNCTIONS OF ONLY TWO ARGUMENTS
}

Nomography 28 deals with the problem: to solve equations by means of drawings of families of curves depending on an arbitrary parameter. It is seen at once that every root of an equation whose coefficients depend upon only two parameters, that is, every function of two independent variables, can be represented in manifold ways according to the principle lying at the foundation of nomography. Further, a large class of functions of three or more variables can evidently be represented by this principle alone without the use of variable elements, namely all those which can be generated by forming first a function of two arguments, then equating each of these arguments to a function of two arguments, next replacing each of those arguments in their turn by a function of two arguments, and so on, regarding as admissible any finite number of insertions of functions of two arguments. So, for example, every rational function of any number of arguments belongs to this class of functions constructed by nomographic tables; for it can be generated by the processes of addition, subtraction, multiplication and division and each of these processes produces a function of only two arguments. One sees easily that the roots of all equations which are solvable by radicals in the natural realm of rationality belong to this class of functions; for here the extraction of roots is adjoined to the four arithmetical operations and this, indeed, presents a function of one argument only. Likewise the general equations of the 5th and 6th degrees are solvable by suitable nomographic tables; for, by means of Tschirnhausen transformations, which require only extraction of roots, they can be reduced to a form where the coefficients depend upon two parameters only.

Now it is probable that the root of the equation of the seventh degree is a function of its coefficients which does not belong to this class of functions capable of nomographic construction, i.e., that it cannot be constructed by a finite number of insertions of functions of two arguments. In order to prove this, the proof would be necessary that the equation of the seventh degree $f^{7}+x f^{3}+y f^{2}+z f+1=0$ is not solvable with the help of any continuous functions of only two arguments. I may be allowed to add that I have satisfied myself by a rigorous process that there exist analytical functions of three arguments $x, y, z$ which cannot be obtained by a finite chain of functions of only two arguments.

By employing auxiliary movable elements, nomography succeeds in constructing functions of more than two arguments, as d'Ocagne has recently proved in the case of the equation of the 7 th degree. 29

\footnotetext{
${ }^{28}$ d'Ocagne, Traité de Nomographie, Paris, 1899.

29 "Sur la résolution nomographique de l'équation du septième degré", Comptes rendus, Paris, 1900.
} 


\section{Proof of the finiteness of Certain COMPlete SYSTEMS OF FUNCTIONS}

In the theory of algebraic invariants, questions as to the finiteness of complete systems of forms deserve, as it seems to me, particular interest. L. Maurer 30 has lately succeeded in extending the theorems on finiteness in invariant theory proved by P. Gordan and myself, to the case where, instead of the general projective group, any subgroup is chosen as the basis for the definition of invariants.

An important step in this direction had been taken already by A. Hurwitz 31 who, by an ingenious process, succeeded in effecting the proof, in its entire generality, of the finiteness of the system of orthogonal invariants of an arbitrary ground form.

The study of the question as to the finiteness of invariants has led me to a simple problem which includes that question as a particular case and whose solution probably requires a decidedly more minutely detailed study of the theory of elimination and of Kronecker's algebraic modular systems than has yet been made.

Let a number $m$ of integral rational functions $X_{1}, X_{2}, \ldots, X_{m}$ for the $n$ variables $x_{1}, x_{2}, \ldots, x_{n}$ be given,

$$
\begin{gathered}
X_{1}=f_{1}\left(x_{1}, \ldots, x_{n}\right), \\
X_{2}=f_{2}\left(x_{1}, \ldots, x_{n}\right), \\
\ldots \ldots \ldots \ldots, \ldots, \ldots, \ldots \\
X_{m}=f_{m}\left(x_{1}, \ldots, x_{n}\right) .
\end{gathered}
$$

Every rational integral combination of $X_{1}, \ldots, X_{m}$ must evidently always become, after substitution of the above expressions, a rational integral function of $x_{1}, \ldots, x_{n}$. Nevertheless, there may well be rational fractional functions of $X_{1}, \ldots, X_{m}$ which, by the operation of the substitution $S$, become integral functions in $x_{1}, \ldots, x_{m}$, which becomes integral in $x_{1}, \ldots, x_{n}$ after the application of the substitution $S, \mathrm{I}$ propose to call a relatively integral function of $X_{1}, \ldots, X_{m}$. Every integral function of $X_{1}, \ldots, X_{m}$ is evidently also relatively integral; further the sum, difference and product of relative integral functions are themselves relatively integral.

The resulting problem is now to decide whether it is always possible to find a finite system of relatively integral function $X_{1}, \ldots, X_{m}$ by which every other relatively integral function of $X_{1}, \ldots, X_{m}$ may be expressed rationally and integrally.

We can formulate the problem still more simply if we introduce the idea of a finite field of integrality. By a finite field of integrality I mean a system of functions from which a finite number of functions can be chosen, in terms of which all other functions of the system are rationally and integrally expressible. Our problem amounts, then, to this: to show that all relatively integral functions of any given domain of rationality always constitute a finite field of integrality.

It naturally occurs to us also to refine the problem by restrictions drawn from number theory, by assuming the coefficients of the given functions $f_{1}, \ldots, f_{m}$ to be integers and including among the relatively integral functions of $X_{1}, \ldots, X_{m}$ only such rational functions of these arguments as become, by the application of the substitutions $S$, rational integral functions of $x_{1}, \ldots, x_{n}$ with rational integral coefficients.

\footnotetext{
${ }^{30}$ Cf. Sitzungsber. d. K. Acad. d. Wiss. zu München, 1899, and an article about to appear in the Math. Annalen.

31 "Ueber die Erzeugung der Invarianten durch Integration", Nachrichten d. K. Gesellschaft d. Wiss. zu Göttingen, 1897.
} 
The following is a simple particular case of this refined problem: Let $m$ integral rational functions $X_{1}, \ldots, X_{m}$ of one variable $x$ with integral rational coefficients, and a prime number $p$ be given. Consider the system of those integral rational functions of $x$ which can be expressed in the form

$$
\frac{G\left(X_{1}, \ldots, X_{m}\right)}{p^{h}},
$$

where $G$ is a rational integral function of the arguments $X_{1}, \ldots, X_{m}$ and $p^{h}$ is any power of the prime number $p$. Earlier investigations of min show immediately that all such expressions for a fixed exponent $h$ form a finite domain of integrality. But the question here is whether the same is true for all exponents $h$, i.e., whether a finite number of such expressions can be chosen by means of which for every exponent $h$ every other expression of that form is integrally and rationally expressible.

From the boundary region between algebra and geometry, I will mention two problems. The one concerns enumerative geometry and the other the topology of algebraic curves and surfaces.

\section{Rigorous Foundation of Schubert's Enumerative CAlCulus}

The problem consists in this: To establish rigorously and with an exact determination of the limits of their validity those geometrical numbers which Schuber 33 especially has determined on the basis of the so-called principle of special position, or conservation of number, by means of the enumerative calculus developed by him.

Although the algebra of to-day guarantees, in principle, the possibility of carrying out the processes of elimination, yet for the proof of the theorems of enumerative geometry decidedly more is requisite, namely, the actual carrying out of the process of elimination in the case of equations of special form in such a way that the degree of the final equations and the multiplicity of their solutions may be foreseen.

\section{Problem of the topology of Algebraic CURVES AND SURFACES}

The maximum number of closed and separate branches which a plane algebraic curve of the $n$th order can have has been determined by Harnack 34 There arises the further question as to the relative position of the branches in the plane. As to curves of the 6th order, I have satisfied myself - by a complicated process, it is true - that of the eleven branches which they can have according to Harnack, by no means all can lie external to one another, but that one branch must exist in whose interior one branch and in whose exterior nine branches lie, or inversely. $A$ thorough investigation of the relative position of the separate branches when their number is the maximum seems to me to be of very great interest, and not less so the corresponding investigation as to the number, form, and position of the sheets of an algebraic surface in space. Till now, indeed, it is not even known what is the maximum number of sheets which a surface of the 4 th order in three dimensional space can really have. 35

\footnotetext{
${ }^{32}$ Math. Annalen, vol. 36 (1890), p. 485.

${ }^{33}$ Kalkül der abzählenden Geometrie, Leipzig, 1879.

${ }^{34}$ Math. Annalen, vol. 10.

${ }^{35}$ Cf. Rohn, "Flächen vierter Ordnung", Preisschriften der Fürstlich Jablonowskischen Gesellschaft, Leipzig, 1886.
} 
In connection with this purely algebraic problem, I wish to bring forward a question which, it seems to me, may be attacked by the same method of continuous variation of coefficients, and whose answer is of corresponding value for the topology of families of curves defined by differential equations. This is the question as to the maximum number and position of Poincaré's boundary cycles (cycles limites) for a differential equation of the first order and degree of the form

$$
\frac{d y}{d x}=\frac{Y}{X}
$$

where $X$ and $Y$ are rational integral functions of the $n$th degree in $x$ and $y$. Written homogeneously, this is

$$
X\left(y \frac{d z}{d t}-z \frac{d y}{d t}\right)+Y\left(z \frac{d x}{d t}-x \frac{d z}{d t}\right)+Z\left(x \frac{d y}{d t}-y \frac{d x}{d t}\right)=0
$$

where $X, Y$, and $Z$ are rational integral homogeneous functions of the $n$th degree in $x, y, z$, and the latter are to be determined as functions of the parameter $t$.

\section{EXPRESSION OF DEFINITE FORMS BY SQUARES}

A rational integral function or form in any number of variables with real coefficients such that it becomes negative for no real values of these variables, is said to be definite. The system of all definite forms is invariant with respect to the operations of addition and multiplication, but the quotient of two definite forms - in case it should be an integral function of the variables - is also a definite form. The square of any form is evidently always a definite form. But since, as I have shown 36 not every definite form can be compounded by addition from squares of forms, the question arises - which I have answered affirmatively for ternary forms 37 whether every definite form may not be expressed as a quotient of sums of squares of forms. At the same time it is desirable, for certain questions as to the possibility of certain geometrical constructions, to know whether the coefficients of the forms to be used in the expression may always be taken from the realm of rationality given by the coefficients of the form represented 38

I mention one more geometrical problem:

\section{BuILDING UP OF SPACE FROM CONGRUENT POLYHEDRA}

If we enquire for those groups of motions in the plane for which a fundamental region exists, we obtain various answers, according as the plane considered is Riemann's (elliptic), Euclid's, or Lobachevsky's (hyperbolic). In the case of the elliptic plane there is a finite number of essentially different kinds of fundamental regions, and a finite number of congruent regions suffices for a complete covering of the whole plane; the group consists indeed of a finite number of motions only. In the case of the hyperbolic plane there is an infinite number of essentially different kinds of fundamental regions, namely, the well-known Poincaré polygons. For the complete covering of the plane an infinite number of congruent regions is necessary. The case of Euclid's plane stands between these; for in this case there is only a

\footnotetext{
${ }^{36}$ Math. Annalen, vol. 32.

${ }^{37}$ Acta Mathematica, vol. 17.

${ }^{38}$ Cf. Hilbert: Grundlagen der Geometrie, Leipzig, 1899, Chap. 7 and in particular $\S 38$.
} 
finite number of essentially different kinds of groups of motions with fundamental regions, but for a complete covering of the whole plane an infinite number of congruent regions is necessary.

Exactly the corresponding facts are found in space of three dimensions. The fact of the finiteness of the groups of motions in elliptic space is an immediate consequence of a fundamental theorem of C. Jordan 39 whereby the number of essentially different kinds of finite groups of linear substitutions in $n$ variables does not surpass a certain finite limit dependent upon $n$. The groups of motions with fundamental regions in hyperbolic space have been investigated by Fricke and Klein in the lectures on the theory of automorphic functions 40 and finally Fedorov 41 Schoenflies 42 and lately Rohn 43 have given the proof that there are, in euclidean space, only a finite number of essentially different kinds of groups of motions with a fundamental region. Now, while the results and methods of proof applicable to elliptic and hyperbolic space hold directly for $n$-dimensional space also, the generalization of the theorem for euclidean space seems to offer decided difficulties. The investigation of the following question is therefore desirable: Is there in $n$ dimensional euclidean space also only a finite number of essentially different kinds of groups of motions with a fundamental region?

A fundamental region of each group of motions, together with the congruent regions arising from the group, evidently fills up space completely. The question arises: Whether polyhedra also exist which do not appear as fundamental regions of groups of motions, by means of which nevertheless by a suitable juxtaposition of congruent copies a complete filling up of all space is possible. I point out the following question, related to the preceding one, and important to number theory and perhaps sometimes useful to physics and chemistry: How can one arrange most densely in space an infinite number of equal solids of given form, e.g., spheres with given radii or regular tetrahedra with given edges (or in prescribed position), that is, how can one so fit them together that the ratio of the filled to the unfilled space may be as great as possible?

If we look over the development of the theory of functions in the last century, we notice above all the fundamental importance of that class of functions which we now designate as analytic functions - a class of functions which will probably stand permanently in the center of mathematical interest.

There are many different standpoints from which we might choose, out of the totality of all conceivable functions, extensive classes worthy of a particularly thorough investigation. Consider, for example, the class of functions characterized by ordinary or partial algebraic differential equations. It should be observed that this class does not contain the functions that arise in number theory and whose investigation is of the greatest importance. For example, the before-mentioned function $\zeta(s)$ satisfies no algebraic differential equation, as is easily seen with the help of the well-known relation between $\zeta(s)$ and $\zeta(1-s)$, if one refers to the theorem proved by

\footnotetext{
${ }^{39}$ Crelle's Journal, vol. 84 (1878), and Atti d. Reale Acad. di Napoli, 1880.

${ }^{40}$ Leipzig, 1897. Cf. especially Abschnitt I, Chapters 2 and 3.

${ }^{41}$ Symmetrie der regelmässigen Systeme von Figuren, 1890.

${ }^{42}$ Krystallsysteme und Krystallstruktur, Leipzig, 1891.

${ }^{43}$ Math. Annalen, vol. 53.
} 
Hölder 44 that the function $\Gamma(x)$ satisfies no algebraic differential equation. Again, the function of the two variables $s$ and $x$ defined by the infinite series

$$
\zeta(s, x)=x+\frac{x^{2}}{2^{s}}+\frac{x^{3}}{3^{s}}+\frac{x^{4}}{4^{s}}+\cdots,
$$

which stands in close relation with the function $\zeta(s)$, probably satisfies no algebraic partial differential equation. In the investigation of this question the functional equation

will have to be used.

$$
x \frac{\partial \zeta(s, x)}{\partial x}=\zeta(s-1, x)
$$

If, on the other hand, we are led by arithmetical or geometrical reasons to consider the class of all those functions which are continuous and indefinitely differentiable, we should be obliged in its investigation to dispense with that pliant instrument, the power series, and with the circumstance that the function is fully determined by the assignment of values in any region, however small. While, therefore, the former limitation of the field of functions was too narrow, the latter seems to me too wide.

The idea of the analytic function on the other hand includes the whole wealth of functions most important to science, whether they have their origin in number theory, in the theory of differential equations or of algebraic functional equations, whether they arise in geometry or in mathematical physics; and, therefore, in the entire realm of functions, the analytic function justly holds undisputed supremacy.

\section{Are the SOlutions of REgular Problems IN THE CALCULUS OF VARIATIONS ALWAYS NECESSARILY ANALYTIC?}

One of the most remarkable facts in the elements of the theory of analytic functions appears to me to be this: That there exist partial differential equations whose integrals are all of necessity analytic functions of the independent variables, that is, in short, equations susceptible of none but analytic solutions. The best known partial differential equations of this kind are the potential equation

$$
\frac{\partial^{2} f}{\partial x^{2}}+\frac{\partial^{2} f}{\partial y^{2}}=0
$$

and certain linear differential equations investigated by Picard 45 also the equation

$$
\frac{\partial^{2} f}{\partial x^{2}}+\frac{\partial^{2} f}{\partial y^{2}}=e^{f}
$$

the partial differential equation of minimal surfaces, and others. Most of these partial differential equations have the common characteristic of being the lagrangian differential equations of certain problems of variation, viz., of such problems of variation

$$
\begin{gathered}
\iint F(p, q, z ; x, y) d x d y=\text { minimum } \\
{\left[p=\frac{\partial z}{\partial x}, q=\frac{\partial z}{\partial y}\right]}
\end{gathered}
$$

\footnotetext{
${ }^{44}$ Math. Annalen, vol. 28.

${ }^{45}$ Jour. de l'Ecole Polytech., 1890.
} 
as satisfy, for all values of the arguments which fall within the range of discussion, the inequality

$$
\frac{\partial^{2} F}{\partial p^{2}} \cdot \frac{\partial^{2} F}{\partial q^{2}}-\left(\frac{\partial^{2} F}{\partial p \partial q}\right)^{2}>0
$$

$F$ itself being an analytic function. We shall call this sort of problem a regular variation problem. It is chiefly the regular variation problems that play a rôle in geometry, in mechanics, and in mathematical physics; and the question naturally arises, whether all solutions of regular variation problems must necessarily be analytic functions. In other words, does every lagrangian partial differential equation of a regular variation problem have the property of admitting analytic integrals exclusively? And is this the case even when the function is constrained to assume, as, e.g., in Dirichlet's problem on the potential function, boundary values which are continuous, but not analytic?

I may add that there exist surfaces of constant negative gaussian curvature which are representable by functions that are continuous and possess indeed all the derivatives, and yet are not analytic; while on the other hand it is probable that every surface whose gaussian curvature is constant and positive is necessarily an analytic surface. And we know that the surfaces of positive constant curvature are most closely related to this regular variation problem: To pass through a closed curve in space a surface of minimal area which shall inclose, in connection with a fixed surface through the same closed curve, a volume of given magnitude.

\section{The General PRoblem of Boundary Values}

An important problem closely connected with the foregoing is the question concerning the existence of solutions of partial differential equations when the values on the boundary of the region are prescribed. This problem is solved in the main by the keen methods of H. A. Schwarz, C. Neumann, and Poincaré for the differential equation of the potential. These methods, however, seem to be generally not capable of direct extension to the case where along the boundary there are prescribed either the differential coefficients or any relations between these and the values of the function. Nor can they be extended immediately to the case where the inquiry is not for potential surfaces but, say, for surfaces of least area, or surfaces of constant positive gaussian curvature, which are to pass through a prescribed twisted curve or to stretch over a given ring surface. It is my conviction that it will be possible to prove these existence theorems by means of a general principle whose nature is indicated by Dirichlet's principle. This general principle will then perhaps enable us to approach the question: Has not every regular variation problem a solution, provided certain assumptions regarding the given boundary conditions are satisfied (say that the functions concerned in these boundary conditions are continuous and have in sections one or more derivatives), and provided also if need be that the notion of a solution shall be suitably extended:46

\footnotetext{
${ }^{46}$ Cf. my lecture on Dirichlet's principle in the Jahresber. d. Deutschen Math.-Vereinigung, vol. 8 (1900), p. 184.
} 


\section{Proof of the EXISTENCE OF LINEAR DIFFERENTIAL EQUATIONS HAVING A PRESCRIBED MONODROMIC GROUP}

In the theory of linear differential equations with one independent variable $z$, I wish to indicate an important problem, one which very likely Riemann himself may have had in mid. This problem is as follows: To show that there always exists a linear differential equation of the Fuchsian class, with given singular points and monodromic group. The problem requires the production of $n$ functions of the variable $z$, regular throughout the complex $z$ plane except at the given singular points; at these points the functions may become infinite of only finite order, and when $z$ describes circuits about these points the functions shall undergo the prescribed linear substitutions. The existence of such differential equations has been shown to be probable by counting the constants, but the rigorous proof has been obtained up to this time only in the particular case where the fundamental equations of the given substitutions have roots all of absolute magnitude unity. L. Schlesinger has given this proof 47 based upon Poincaré's theory of the Fuchsian $\zeta$-functions. The theory of linear differential equations would evidently have a more finished appearance if the problem here sketched could be disposed of by some perfectly general method.

\section{UNIFORMIZATION OF ANALYTIC RELATIONS BY MEANS OF AUTOMORPHIC FUNCTIONS}

As Poincaré was the first to prove, it is always possible to reduce any algebraic relation between two variables to uniformity by the use of automorphic functions of one variable. That is, if any algebraic equation in two variables be given, there can always be found for these variables two such single valued automorphic functions of a single variable that their substitution renders the given algebraic equation an identity. The generalization of this fundamental theorem to any analytic nonalgebraic relations whatever between two variables has likewise been attempted with success by Poincaré 48 though by a way entirely different from that which served him in the special problem first mentioned. From Poincaré's proof of the possibility of reducing to uniformity an arbitrary analytic relation between two variables, however, it does not become apparent whether the resolving functions can be determined to meet certain additional conditions. Namely, it is not shown whether the two single valued functions of the one new variable can be so chosen that, while this variable traverses the regular domain of those functions, the totality of all regular points of the given analytic field are actually reached and represented. On the contrary it seems to be the case, from Poincaré's investigations, that there are beside the branch points certain others, in general infinitely many other discrete exceptional points of the analytic field, that can be reached only by making the new variable approach certain limiting points of the functions. In view of the fundamental importance of Poincarés formulation of the question it seems to me that an elucidation and resolution of this difficulty is extremely desirable.

In conjunction with this problem comes up the problem of reducing to uniformity an algebraic or any other analytic relation among three or more complex variablesa problem which is known to be solvable in many particular cases. Toward the

\footnotetext{
${ }^{47}$ Handbuch der Theorie der linearen Differentialgleichungen, vol. 2, part 2, No. 366.

${ }^{48}$ Bull. de la Soc. Math. de France, vol. 11 (1883).
} 
solution of this the recent investigations of Picard on algebraic functions of two variables are to be regarded as welcome and important preliminary studies.

\section{FURTHER DEVELOPMENT OF THE METHODS OF THE CALCULUS OF VARIATIONS}

So far, I have generally mentioned problems as definite and special as possible, in the opinion that it is just such definite and special problems that attract us the most and from which the most lasting influence is often exerted upon science. Nevertheless, I should like to close with a general problem, namely with the indication of a branch of mathematics repeatedly mentioned in this lecture - which, in spite of the considerable advancement lately given it by Weierstrass, does not receive the general appreciation which, in my opinion, is its due - I mean the calculus of variations 49

The lack of interest in this is perhaps due in part to the need of reliable modern text-books. So much the more praiseworthy is it that A. Kneser in a very recently published work has treated the calculus of variations from the modern points of view and with regard to the modern demand for rigor 50

The calculus of variations is, in the widest sense, the theory of the variation of functions, and as such appears as a necessary extension of the differential and integral calculus. In this sense, Poincaré's investigations on the problem of three bodies, for example, form a chapter in the calculus of variations, in so far as Poincaré derives from known orbits by the principle of variation new orbits of similar character.

I add here a short justification of the general remarks upon the calculus of variations made at the beginning of my lecture.

The simplest problem in the calculus of variations proper is known to consist in finding a function $y$ of a variable $x$ such that the definite integral

$$
J=\int_{a}^{b} F\left(y_{x}, y ; x\right) d x, \quad y_{x}=\frac{d y}{d x}
$$

assumes a minimum value as compared with the values it takes when $y$ is replaced by other functions of $x$ with the same initial and final values.

The vanishing of the first variation in the usual sense

$$
\delta J=0
$$

gives for the desired function $y$ the well-known differential equation

$$
\begin{gathered}
\frac{d F_{y_{x}}}{d x}-F_{y}=0 \\
{\left[F_{y_{x}}=\frac{\partial F}{\partial y_{x}}, \quad F_{y}=\frac{\partial F}{\partial y}\right] .}
\end{gathered}
$$

\footnotetext{
${ }^{49}$ Text-books: Moigno-Lindelöf, Leçons du calcul des variations, Paris, 1861, and A. Kneser, Lehrbuch der Variations-rechnung, Braunschweig, 1900.

${ }^{50} \mathrm{As}$ an indication of the contents of this work, it may here be noted that for the simplest problems Kneser derives sufficient conditions of the extreme even for the case that one limit of integration is variable, and employs the envelope of a family of curves satisfying the differential equations of the problem to prove the necessity of Jacobi's conditions of the extreme. Moreover, it should be noticed that Kneser applies Weierstrass's theory also to the inquiry for the extreme of such quantities as are defined by differential equations.
} 
In order to investigate more closely the necessary and sufficient criteria for the occurrence of the required minimum, we consider the integral

$$
\begin{gathered}
J^{*}=\int_{a}^{b}\left\{F+\left(y_{x}-p\right) F_{p}\right\} d x, \\
\left.F=F(p, y ; x), F_{p}=\frac{\partial F(p, y ; x)}{\partial p}\right] .
\end{gathered}
$$

Now we inquire how $p$ is to be chosen as function of $x, y$ in order that the value of this integral $J^{*}$ shall be independent of the path of integration, i.e., of the choice of the function $y$ of the variable $x$. The integral $J^{*}$ has the form

$$
J^{*}=\int_{a}^{b}\left\{A y_{x}-B\right\} d x,
$$

where $A$ and $B$ do not contain $y_{x}$ and the vanishing of the first variation

$$
\delta J^{*}=0
$$

in the sense which the new question requires gives the equation

$$
\frac{\partial A}{\partial x}+\frac{\partial B}{\partial y}=0
$$

i.e., we obtain for the function $p$ of the two variables $x, y$ the partial differential equation of the first order

$$
\frac{\partial F_{p}}{\partial x}+\frac{\partial\left(p F_{p}-F\right)}{\partial y}=0 .
$$

The ordinary differential equation of the second order (1) and the partial differential equation $\left(1^{*}\right)$ stand in the closest relation to each other. This relation becomes immediately clear to us by the following simple transformation

$$
\begin{aligned}
\delta J^{*} & =\int_{a}^{b}\left\{F_{y} \delta y+F_{p} \delta p+\left(\delta y_{x}-\delta p\right) F_{y}+\left(y_{x}-p\right) \delta F_{p}\right\} d x \\
& =\int_{a}^{b}\left\{F_{y} \delta y+\delta y_{x} F_{p}+\left(y_{x}-p\right) \delta F_{p}\right\} d x \\
& =\delta J+\int_{a}^{b}\left(y_{x}-p\right) \delta F_{p} d x .
\end{aligned}
$$

We derive from this, namely, the following facts: If we construct any simple family of integral curves of the ordinary differential equation (1) of the second order and then form an ordinary differential equation of the first order

$$
y_{x}=p(x, y)
$$

which also admits these integral curves as solutions, then the function $p(x, y)$ is always an integral of the partial differential equation $\left(1^{*}\right)$ of the first order; and conversely, if $p(x, y)$ denotes any solution of the partial differential equation $\left(1^{*}\right)$ of the first order, all the non-singular integrals of the ordinary differential equation (2) of the first order are at the same time integrals of the differential equation (1) of the second order, or in short if $y_{x}=p(x, y)$ is an integral equation of the first order of the differential equation (1) of the second order, $p(x, y)$ represents an integral of the partial differential equation $\left(1^{*}\right)$ and conversely; the integral curves of the ordinary differential equation of the second order are therefore, at the same time, the characteristics of the partial differential equation $\left(1^{*}\right)$ of the first order. 
In the present case we may find the same result by means of a simple calculation; for this gives us the differential equations (1) and $\left(1^{*}\right)$ in question in the form

$$
\begin{aligned}
& y_{x x} F_{y_{x} y_{x}}+y_{x} F_{y_{x} y}+F_{y_{x} x}-F_{y}=0, \\
& \left(p_{x}+p p_{y}\right) F_{p p}+p F_{p y}+F_{p x}-F_{y}=0,
\end{aligned}
$$

where the lower indices indicate the partial derivatives with respect to $x, y, p, y_{x}$. The correctness of the affirmed relation is clear from this.

The close relation derived before and just proved between the ordinary differential equation (1) of the second order and the partial differential equation $\left(1^{*}\right)$ of the first order, is, as it seems to me, of fundamental significance for the calculus of variations. For, from the fact that the integral $J^{*}$ is independent of the path of integration it follows that

$$
\int_{a}^{b}\left\{F(p)+\left(y_{x}-p\right) F_{p}(p)\right\} d x=\int_{a}^{b} F\left(\bar{y}_{x}\right) d x,
$$

if we think of the left hand integral as taken along any path $y$ and the right hand integral along an integral curve $\bar{y}$ of the differential equation

$$
\bar{y}_{x}=p(x, \bar{y}) \text {. }
$$

With the help of equation (3) we arrive at Weierstrass's formula

$$
\int_{a}^{b} F\left(y_{x}\right) d x-\int_{a}^{b} F\left(\bar{y}_{x}\right) d x=\int_{a}^{b} E\left(y_{x}, p\right) d x
$$

where $E$ designates Weierstrass's expression, depending upon $y_{x}, p, y, x$,

$$
E\left(y_{x}, p\right)=F\left(y_{x}\right)-F(p)-\left(y_{x}-p\right) F_{p}(p) .
$$

Since, therefore, the solution depends only on finding an integral $p(x, y)$ which is single valued and continuous in a certain neighborhood of the integral curve $\bar{y}$, which we are considering, the developments just indicated lead immediatelywithout the introduction of the second variation, but only by the application of the polar process to the differential equation (1) - to the expression of Jacobi's condition and to the answer to the question: How far this condition of Jacobi's in conjunction with Weierstrass's condition $E>0$ is necessary and sufficient for the occurrence of a minimum.

The developments indicated may be transferred without necessitating further calculation to the case of two or more required functions, and also to the case of a double or a multiple integral. So, for example, in the case of a double integral

$$
J=\int F\left(z_{x}, z_{y}, z ; x, y\right) d \omega, \quad\left[z_{x}=\frac{\partial z}{\partial x}, \quad z_{y}=\frac{\partial z}{\partial y}\right]
$$

to be extended over a given region $\omega$, the vanishing of the first variation (to be understood in the usual sense)

$$
\delta J=0
$$

gives the well-known differential equation of the second order

$$
\begin{gathered}
\frac{d F_{z}}{d x}+\frac{d F_{z_{y}}}{d y}-F_{z}=0 \\
{\left[F_{z_{x}}=\frac{\partial F}{\partial z}, F_{z}=\frac{\partial F}{\partial z_{y}}, F_{z}-\frac{\partial F}{\partial z}\right]}
\end{gathered}
$$


for the required function $z$ of $x$ and $y$.

On the other hand we consider the integral

$$
\begin{gathered}
J^{*}=\int\left\{F+\left(z_{x}-p\right) F_{p}+\left(z_{y}-q\right) F_{q}\right\} d \omega, \\
{\left[F=F(p, q, z ; x, y), F_{p}=\frac{\partial F(p, q, z ; x, y)}{\partial p}, F_{q}=\frac{\partial F(p, q, z ; x, y)}{\partial q}\right],}
\end{gathered}
$$

and inquire, how $p$ and $q$ are to be taken as functions of $x, y$ and $z$ in order that the value of this integral may be independent of the choice of the surface passing through the given closed twisted curve, i.e., of the choice of the function $z$ of the variables $x$ and $y$.

The integral $J^{*}$ has the form

$$
J^{*}=\int\left\{A z_{x}+B z_{y}-C\right\} d \omega
$$

and the vanishing of the first variation

$$
\delta J^{*}=0,
$$

in the sense which the new formulation of the question demands, gives the equation

$$
\frac{\partial A}{\partial x}+\frac{\partial B}{\partial y}+\frac{\partial C}{\partial z}=0
$$

i.e., we find for the functions $p$ and $q$ of the three variables $x, y$ and $z$ the differential equation of the first order

$$
\frac{\partial F_{p}}{\partial x}+\frac{\partial F_{q}}{\partial y}+\frac{\partial\left(p F_{p}+q F_{q}-F\right)}{\partial x}=0 .
$$

If we add to this differential equation the partial differential equation

$$
p_{y}+q p_{z}=q_{x}+p q_{z}
$$

resulting from the equations

$$
z_{x}=p(x, y, z), \quad z_{y}=q(x, y, z),
$$

the partial differential equation (I) for the function $z$ of the two variables $x$ and $y$ and the simultaneous system of the two partial differential equations of the first order $\left(\mathrm{I}^{*}\right)$ for the two functions $p$ and $q$ of the three variables $x, y$, and $z$ stand toward one another in a relation exactly analogous to that in which the differential equations (1) and $\left(1^{*}\right)$ stood in the case of the simple integral.

It follows from the fact that the integral $J^{*}$ is independent of the choice of the surface of integration $z$ that

$$
\begin{gathered}
\int\left\{F(p, q)+\left(z_{x}-p\right) F_{p}(p, q)+\left(z_{y}-q\right) F_{q}(p, q)\right\} d \omega \\
=\int F\left(\bar{z}_{x}, \bar{y}\right) d \omega,
\end{gathered}
$$

if we think of the right hand integral as taken over an integral surface $\bar{z}$ of the partial differential equations

$$
\bar{z}_{x}=p(x, y, \bar{z}), \bar{z}_{y}=q(x, y, \bar{z})
$$


and with the help of this formula we arrive at once at the formula

$$
\begin{gathered}
\int F\left(z_{x}, z_{y}\right) d \omega-\int F\left(\bar{z}_{x}, \bar{z}_{y}\right) d \omega=\int E\left(z_{x}, z_{y}, p, q\right) d \omega \\
{\left[E\left(z_{x}, z_{y}, p, q\right)=F\left(z_{x}, z_{y}\right)-F(p, q)-\left(z_{x}-p\right) F_{p}(p, q)\right.} \\
\left.-\left(z_{y}-q\right) F_{q}(p, q)\right],
\end{gathered}
$$

which plays the same rôle for the variation of double integrals as the previously given formula (4) for simple integrals. With the help of this formula we can now answer the question how far Jacobi's condition in conjunction with Weierstrass's condition $E>0$ is necessary and sufficient for the occurrence of a minimum.

Connected with these developments is the modified form in which A. Kneser 51 beginning from other points of view, has presented Weierstrass's theory. While Weierstrass employed to derive sufficient conditions for the extreme tehos integral curves of equation (1) which pass through a fixed point, Kneser on the other hand makes use of any simple family of such curves and constructs for every such family a solution, characteristic for that family, of that partial differential equation which is to be considered as a generalization of the Jacobi-Hamilton equation.

The problems mentioned are merely samples of problems, yet they will suffice to show how rich, how manifold and how extensive the mathematical science of to-day is, and the question is urged upon us whether mathematics is doomed to the fate of those other sciences that have split up into separate branches, whose representatives scarcely understand one another and whose connection becomes ever more loose. I do not believe this nor wish it. Mathematical science is in my opinion an indivisible whole, an organism whose vitality is conditioned upon the connection of its parts. For with all the variety of mathematical knowledge, we are still clearly conscious of the similarity of the logical devices, the relationship of the ideas in mathematics as a whole and the numerous analogies in its different departments. We also notice that, the farther a mathematical theory is developed, the more harmoniously and uniformly does its construction proceed, and unsuspected relations are disclosed between hitherto separate branches of the science. So it happens that, with the extension of mathematics, its organic character is not lost but only manifests itself the more clearly.

But, we ask, with the extension of mathematical knowledge will it not finally become impossible for the single investigator to embrace all departments of this knowledge? In answer let me point out how thoroughly it is ingrained in mathematical science that every real advance goes hand in hand with the invention of sharper tools and simpler methods which at the same time assist in understanding earlier theories and cast aside older more complicated developments. It is therefore possible for the individual investigator, when he makes these sharper tools and simpler methods his own, to find his way more easily in the various branches of mathematics than is possible in any other science.

The organic unity of mathematics is inherent in the nature of this science, for mathematics is the foundation of all exact knowledge of natural phenomena. That it may completely fulfil this high mission, may the new century bring it gifted masters and many zealous and enthusiastic disciples.

${ }^{51}$ Cf. his above-mentioned textbook, $\S \S 14,15,19$ and 20. 